\title{
Absolute stability and Lyapunov-Krasovskii functionals for switched nonlinear systems with time-delay
}

\author{
A.Yu. Aleksandrov ${ }^{\mathrm{b}, 2}$, Oliver Mason ${ }^{\mathrm{a}, *, 1}$ \\ ${ }^{\mathrm{a}}$ Hamilton Institute, National University of Ireland Maynooth, Co. Kildare, Ireland \\ ${ }^{\mathrm{b}}$ Faculty of Applied Mathematics and Control Processes, St. Petersburg State University, St. Petersburg, Russia
}

Received 20 December 2013; received in revised form 30 April 2014; accepted 24 May 2014

Available online 2 June 2014

\begin{abstract}
We derive conditions for the existence of Lyapunov-Krasovskii functionals for a number of classes of nonlinear switched systems with time-delay. In particular, we first give conditions for systems of retarded type that relax those recently described in Sun and Wang (2013) [18]. Using similar techniques, related results are then derived for coupled differential-difference systems and for systems of neutral type. Finally, we briefly note some corresponding results for discrete-time systems.

(c) 2014 The Franklin Institute. Published by Elsevier Ltd. All rights reserved.
\end{abstract}

\section{Introduction}

Lyapunov-Krasovskii (L-K) functionals are a key tool in the stability theory of time-delayed dynamical systems [1]. In the search for $\mathrm{L}-\mathrm{K}$ functionals, researchers typically focus on a particular form and then seek conditions for the existence of functionals of this form [2,3]. Over recent years, there has been considerable interest in the analysis of the so-called positive systems $[4,5]$; the defining characteristic of such systems being that the nonnegative orthant is invariant under the dynamics of the system. The interest in positive systems stems from their appealing theoretical properties coupled with their practical importance in fields such as Economics,

\footnotetext{
*Corresponding author. Tel.: +3531 7086274; fax: +3531 7086269.

E-mail address: oliver.mason@nuim.ie (O. Mason).

${ }^{1}$ Supported by the Irish Higher Educational Authority (HEA) PRTLI 4 Network Mathematics Grant.

${ }^{2}$ Supported by the St. Petersburg State University, Project no. 9.38.674.2013, and by the Russian Foundation of Basic Researches (RFBR), Grant nos. 13-01-00376-a and 13-08-00948-a.
} 
Demography and Biology. Their defining property allows the use of linear Lyapunov functions and functionals [6-8] as the functional need only be positive definite on the nonnegative orthant. Moreover, using comparison theorems, stability conditions derived for positive systems can be used to establish conditions for related systems that are not necessarily positive. This last remark applies to the results of this paper.

Our primary goal in this paper is to build on the previous work on linear Lyapunov functions and functionals $[9,6,13,14]$ for positive systems and to derive conditions for the existence of such functionals for classes of nonlinear time-delay systems. Many of our results pertain to switched systems and there is an extensive literature on the stability of such systems $[9,13,6,10-12]$. In broad terms, we give results for three classes of systems. We first consider switched nonlinear positive systems of the form recently studied in [18]; we give a condition for absolute stability and the existence of linear L-K functionals for this class that relaxes the result in [18]. Building on this, we show that similar techniques can be deployed to derive conditions for linear $\mathrm{L}-\mathrm{K}$ functional existence for switched coupled differential-difference equations [21] and switched neutral systems. Systems described by coupled differential and difference equations arise in applications such as hydraulics, circuit theory, and the analysis of partial differential equations. They can also be used to reduce the dimensionality of a large-scale delayed system, by suitably exploiting the fact that, while an overall system may be high-dimensional, delays often occur in scalar or low-dimensional parts of the system [20]. Neutral systems in which delays appear in the derivative of the state vector as well as in state itself arise in a number of applications and have been widely studied [1]. These results underline the broad applicability of linear L-K functionals.

The layout of the paper is as follows. In the next section, we define the main notation used throughout the paper and provide the mathematical background and context for our results. In Section 3, we present our result for switched nonlinear systems that relaxes the conditions given in [18]. In Section 4, we turn our attention to coupled differential-difference equations, while the absolute stability of neutral systems is considered in Section 5. Finally, in Sections 6 and 7 we note that similar results naturally hold for discrete time systems and present our conclusions.

\section{Context and background}

We denote the space of continuous functions from $[-\tau, 0]$ to $\mathbb{R}^{n}$ by $C\left([-\tau, 0], \mathbb{R}^{n}\right)$. A matrix $A \in \mathbb{R}^{n \times n}$ is Hurwitz if all of its eigenvalues have negative real part. $A$ is said to be Schur-Cohn if all of its eigenvalues have modules less than one. We use $e$ to denote the vector all of whose entries are equal to one, $e=(1, \ldots, 1)^{T}$. For a vector $v \in \mathbb{R}^{n}, v \geq 0$ means that $v_{i} \geq 0$ for $1 \leq i \leq n$. Similarly, $v>0$ means that $v \geq 0, v \neq 0$, while $v \gg 0$ means that $v_{i}>0$ for all $i$. For a vector $x \in \mathbb{R}^{n}$, the notation $|x|$ denotes the vector $\left(\left|x_{1}\right|, \ldots,\left|x_{n}\right|\right)^{T}$. For a functional $V$ defined on $C\left([-\tau, 0], \mathbb{R}^{n}\right), \partial^{+} V$ denotes the upper-right Dini derivative of $V[1]$.

The use of linear Lyapunov functions to study positive and related systems stretches back beyond the interest of the last decade. We here recall various system classes to which these have been applied, with the complexity increasing as we proceed. In [15], the system

$$
\dot{x}(t)=A f(x(t))
$$

is considered. The nonlinearity $f: \mathbb{R}^{n} \rightarrow \mathbb{R}^{n}$ is continuous, diagonal

$$
f\left(x_{1}, \ldots, x_{n}\right)=\left(f_{1}\left(x_{1}\right), f_{2}\left(x_{2}\right), \ldots, f_{n}\left(x_{n}\right)\right)^{T}
$$


and assumed to satisfy

$$
x_{i} f_{i}\left(x_{i}\right)>0 \text { for } x_{i} \neq 0 .
$$

Note that this implies that $f_{i}\left(x_{i}\right)<0$ for $x_{i}<0, f_{i}\left(x_{i}\right)>0$ for $x_{i}>0$; it now follows immediately from continuity that $f_{i}(0)=0$ for $1 \leq i \leq n$. Such a nonlinearity is said to be admissible.

The matrix $A$ is assumed to be Metzler, meaning that its off-diagonal elements $a_{i j}, i \neq j$ are nonnegative. Note that, if the nonlinearity satisfies an appropriate Lipschitz condition so that solutions of Eq. (1) are unique, then these conditions mean that Eq. (1) is a positive system with an equilibrium at the origin, see [15].

It is established in [15] that if $A$ is a Hurwitz matrix then the zero solution of Eq. (1) is asymptotically stable for any admissible nonlinearity $f(x)$, and a Lyapunov function can be chosen in the form

$$
V(x)=\nu^{T}|x|=\sum_{i=1}^{n} \nu_{i}\left|x_{i}\right|
$$

where the positive vector $\nu=\left(\nu_{1}, \ldots, \nu_{n}\right)^{T}$ is a solution of the system $A^{T} \nu \ll 0$.

Moving to delayed systems, in [16] linear L-K functionals were used to analyse

$$
\dot{x}(t)=A x(t)+B x(t-\tau) .
$$

where $A$ is Metzler and $B$ is nonnegative. This guarantees that the system is positive. In [16] it is shown that the zero solution of this system is asymptotically stable for all $\tau \geq 0$ if and only if there is some vector $\nu \gg 0$ with $(A+B)^{T} \nu \ll 0$. In this case, a $\mathrm{L}-\mathrm{K}$ functional of the form

$$
V\left(x_{t}\right)=\nu^{T}|x(t)|+\int_{t-\tau}^{t} \nu^{T} B|x(\theta)| d \theta
$$

exists. As usual $x_{t}$ denotes the continuous function on $[-\tau, 0]$ given by $x_{t}(\theta)=x(t+\theta)$ for $\theta \in[-\tau, 0]$. Where there is no risk of confusion, we shall refer to the stability of the system rather than of the zero solution.

An extension of the core result of [15] to switched systems was given in [9] (related results can be found in [17]). This paper considers a switched system $\dot{x}(t)=A^{(\sigma)} f(x(t))$ with a corresponding family of constituent systems $\dot{x}(t)=A^{(s)} f(x(t)), s=1, \ldots, N$ where $A^{(s)}$ are Metzler and Hurwitz matrices, and the diagonal nonlinearity $f: \mathbb{R}^{n} \rightarrow \mathbb{R}^{n}$ satisfies the condition (2). Extending the result of [15], it is shown that if there exists a positive vector $\nu \gg 0$ satisfying $A^{(s)^{T}} \nu \ll 0, s=1, \ldots, N$, then the nonlinear switched system is asymptotically stable for any admissible nonlinearity $f(x)$ and for arbitrary switching signals. Moreover, a common Lyapunov function can be chosen in the form $\nu^{T}|x|$. In the next section, we consider a class of switched nonlinear system with time-delay.

\section{Switched nonlinear differential systems with time-delay}

The system

$$
\dot{x}(t)=A^{(\sigma)} f(x(t))+B^{(\sigma)} f(x(t-\tau))
$$

is considered in [18]. The corresponding family of subsystems is given by

$$
\dot{x}(t)=A^{(s)} f(x(t))+B^{(s)} f(x(t-\tau)), \quad s=1, \ldots, N .
$$

Here $A^{(s)}$ are Metzler and Hurwitz matrices, $B^{(s)}$ are nonnegative matrices, and the diagonal nonlinearity $f: \mathbb{R}^{n} \rightarrow \mathbb{R}^{n}$ is continuous and satisfies Eq. (2). 
Throughout the paper, an admissible switching signal is a piecewise constant function $\sigma:[0, \infty) \rightarrow\{1, \ldots, N\}$, which is right continuous and has only finitely many discontinuities on every bounded interval.

We say that Eq. (3) is absolutely stable if the origin is a globally asymptotically stable equilibrium for every admissible switching signal $\sigma$, every continuous diagonal nonlinearity satisfying Eq. (2) and every nonnegative delay $\tau$. For a non-switched system, absolute stability is defined with respect to the family of nonlinearities and all nonnegative delays only.

Let $b_{i j}^{(s)}$ denote the entries of $B^{(s)}$, and write $\bar{B}=\left\{\bar{b}_{i j}\right\}$, where

$$
\bar{b}_{i j}=\max _{s=1, \ldots, N} b_{i j}^{(s)} .
$$

In [18], it was proved that if there exists a positive vector $\nu=\left(\nu_{1}, \ldots, \nu_{n}\right)^{T}$ such that $\left(A^{(s)}+\bar{B}\right)^{T} \nu \ll 0$ for $s=1, \ldots, N$, then the switched system (3) is absolutely stable, and a common $\mathrm{L}-\mathrm{K}$ functional can be chosen in the form

$$
V\left(x_{t}\right)=\sum_{i=1}^{n} \nu_{i}\left|x_{i}(t)\right|+\sum_{i=1}^{n} \nu_{i} \int_{t-\tau}^{t} \sum_{j=1}^{n} \bar{b}_{i j}\left|f_{j}\left(x_{j}(\theta)\right)\right| d \theta .
$$

Our goal is to obtain less restrictive stability conditions for Eq. (3) than those in [18].

To begin, we consider the single delay case in the interest of notational simplicity and clarity; the arguments for the several delay cases are identical.

\subsection{A single delay}

Theorem 3.1. Consider the system (3). Assume that $A^{(s)}$ is Metzler and $B^{(s)}$ is nonnegative for $s=1, \ldots, N$. Assume that there exists a vector $\nu \gg 0$ satisfying the inequalities

$$
\left(A^{(s)}+B^{(r)}\right)^{T} \nu \ll 0, \quad s, r=1, \ldots, N .
$$

Then, the switched system (3) is absolutely stable; in fact, there exist positive constants $\mu_{1}, \ldots, \mu_{n}$ such that

$$
V=\sum_{i=1}^{n} \nu_{i}\left|x_{i}(t)\right|+\sum_{i=1}^{n} \mu_{i} \int_{t-\tau}^{t}\left|f_{i}\left(x_{i}(z)\right)\right| d z
$$

defines a $L-K$ functional for the family (3).

Proof. By assumption, there exists some vector $\nu \gg 0$ with $\left(A^{(s)}+B^{(r)}\right)^{T} \nu \ll 0$ for all $s, r$. Choose some positive vector $q \gg 0$ such that

$$
\left(A^{(s)}+B^{(r)}\right)^{T} \nu \leq-q .
$$

Next set $w=\max \left\{\left(B^{(s)}\right)^{T} \nu\right\}$, where the maximum is taken elementwise. Clearly $w \geq 0$. Now choose $\mu=w+q / 2$. It follows readily from Eq. (7) that for all $s \in\{1, \ldots, N\}$,

$$
\left(A^{(s)}\right)^{T} \nu+w \leq-q
$$

so that

$$
\left(A^{(s)}\right)^{T} \nu \leq-q-w \ll-\mu .
$$

On the other hand, it is immediate from the definition of $w$ that for all $s \in\{1, \ldots, N\}$,

$$
\left(B^{(s)}\right)^{T} \nu \ll \mu \text {. }
$$


If we differentiate Eq. (6) along any of the systems (4) we find that the derivative satisfies

$$
\partial^{+} V \leq|f(x(t))|^{T}\left(A^{(s)^{T}} \nu+\mu\right)+|f(x(t-\tau))|^{T}\left(B^{(s)^{T}} \nu-\mu\right) \leq-\beta \sum_{j=1}^{n}\left|f_{j}\left(x_{j}(t)\right)\right|,
$$

where $\beta$ is a positive constant. Thus $V$ defines a $\mathrm{L}-\mathrm{K}$ functional for Eq. (3) as claimed. As this is true for any choice of $f$ and $\tau$, it follows that Eq. (3) is absolutely stable as claimed.

\section{Remarks.}

(i) The results of [18] can be applied to Eq. (3) to provide a closely related set of conditions for absolute stability. While this previous paper does not restrict the system matrices to be Metzler and nonnegative, the authors provide stability conditions in terms of comparison matrices that are Metzler and nonnegative. Our above result can be applied to more general systems in the same way via comparison theorems.

(ii) It is important to note some key distinctions between our work and the result in [18]. First of all, the L-K functional that we use is diagonal in contrast to those in [18] and [16], where the upper bound matrix $\bar{B}$ and the full delay matrix $B$ are used in the integral term. As discussed extensively in [22], diagonal type functions and functionals have many appealing properties in addition to their simplicity. It is more important to note that our result gives less restrictive conditions than those in [18]. The intuitive reason for this is that the $\bar{B}$ matrix describes an upper bound or a worst case scenario that does not necessarily correspond to the dynamics of any of the constituent systems. Essentially, by using $\bar{B}$ to provide a stability condition, we sacrifice system information; our approach uses only the matrices defining the dynamics of the system to construct the $\mathrm{L}-\mathrm{K}$ functional and for this reason provides less conservative conditions. The numerical example given below illustrates this last point.

(iii) While our result provides a less restrictive condition for stability, it does come with a higher computational cost. The condition in [18] requires the checking of $N$ linear inequalities, while ours involves $N^{2}$. The generalisation to multiple delays is given in Theorem 3.2 and the computational burden increases with the number of delays and constituent systems. In general, if we have $N$ constituent systems and $l$ delays, the number of inequalities to be checked is $N^{l+1}$. It is worth pointing out however that with modern software for linear programming, a problem involving several thousand inequalities is very tractable.

Example 3.1. Consider an example of the system (3) with $n=2, N=2$ and

$$
\begin{aligned}
& A=A_{1}=A_{2}=\left(\begin{array}{cc}
-2 & 0 \\
0 & -2
\end{array}\right), \\
& B_{1}=\left(\begin{array}{ll}
1 & 1 \\
1 & 0
\end{array}\right), \quad B_{2}=\left(\begin{array}{ll}
0 & 1 \\
3 & 0
\end{array}\right) .
\end{aligned}
$$

On the one hand, if we apply the approach from [18], we would first construct the matrix

$$
\bar{B}=\left(\begin{array}{ll}
1 & 1 \\
3 & 0
\end{array}\right)
$$

which is the entrywise maximum of $B_{1}$ and $B_{2}$. In this case, it can be easily verified that there is no $\nu \gg 0$ such that $(A+\bar{B})^{T} \nu \ll 0$. Hence, we cannot apply Theorem 2 of [18] in this case. 
On the other hand, applying Theorem 3.1, we can check that

$$
\left(A+B^{(s)}\right)^{T} \nu \ll 0, \quad s=1,2,
$$

with $\nu=(7,4)^{T}$ for example.

Remark. It is worth mentioning that the existence of a common Lyapunov function in the form

$$
V(x)=\nu^{T}|x|
$$

for the corresponding delay free system does not necessarily imply the existence of a common $\mathrm{L}-\mathrm{K}$ functional in the form (6) for the system (3).

Example 3.2. Consider the simple 1-dimensional systems

$$
\begin{aligned}
& \dot{x}(t)=-0.2 x(t)+0.1 x(t-\tau), \\
& \dot{x}(t)=-0.9 x(t)+0.8 x(t-\tau) .
\end{aligned}
$$

As the undelayed systems obtained by setting the delay to zero are identical, they clearly have a common Lyapunov function of the required form. However, it can be easily verified that no common L-K functional in the form (6) exists for the switched system. In fact, for such a functional to exist, we would require that $0.1 \nu<\mu<0.2 \nu$ and $0.8 \nu<\mu<0.9 \nu$, which is clearly impossible.

\subsection{Several delays}

Finally, for this section, consider the switched system with several delays

$$
\dot{x}(t)=A^{(\sigma)} f(x(t))+B_{1}^{(\sigma)} f\left(x\left(t-\tau_{1}\right)\right)+\cdots+B_{l}^{(\sigma)} f\left(x\left(t-\tau_{l}\right)\right)
$$

and the corresponding family of subsystems

$$
\dot{x}(t)=A^{(s)} f(x(t))+B_{1}^{(s)} f\left(x\left(t-\tau_{1}\right)\right)+\cdots+B_{l}^{(s)} f\left(x\left(t-\tau_{l}\right)\right), \quad s=1, \ldots, N,
$$

where $A^{(s)}$ are Metzler matrices, and $B_{1}^{(s)}, \ldots, B_{l}^{(s)}$ are nonnegative matrices for $1 \leq s \leq N$, and the nonlinearities $f(x)=\left(f_{1}\left(x_{1}\right), \ldots, f_{n}\left(x_{n}\right)\right)^{T}$ are continuous and satisfy the condition (2). The real numbers $\tau_{1}, \ldots, \tau_{l}$ are nonnegative delays.

It is relatively straightforward to adapt the argument used in the proof of Theorem 2.1 to obtain the following result for systems with several delays.

Theorem 3.2. Assume that there exists a positive vector $\nu \gg 0$ satisfying the inequalities

$$
\left(A^{(s)}+B_{1}^{\left(p_{1}\right)}+\cdots+B_{l}^{\left(p_{l}\right)}\right)^{T} \nu \ll 0,
$$

for $s, p_{1}, \ldots, p_{l} \in\{1, \ldots, N\}$. Then the switched system (10) is absolutely stable, and for the family (11) there exist positive constants $\mu_{r j}$ for $r=1, \ldots, l, j=1, \ldots, n$ such that

$$
V=\sum_{i=1}^{n} \nu_{i}\left|x_{i}(t)\right|+\sum_{i=1}^{n} \sum_{r=1}^{l} \int_{t-\tau_{r}}^{t} \mu_{r i}\left|f_{i}\left(x_{i}(z)\right)\right| d z
$$

is a common $L-K$ functional for the system (10).

Remark. To check for the existence of a positive vector $\nu \gg 0$ satisfying the inequalities (12) and to find this vector, one can apply approaches proposed in $[6,17,19]$. 


\section{Coupled nonlinear differential and difference systems}

In this section, we present sufficient conditions for absolute stability of a class of nonlinear coupled differential-difference systems using a L-K functional of linear form.

\subsection{Nonswitched systems}

To begin, consider the system

$$
\begin{aligned}
& \dot{x}(t)=A f(x(t))+B y(t-\tau), \\
& y(t)=C f(x(t))+D y(t-\tau) .
\end{aligned}
$$

Here $x(t) \in \mathbb{R}^{n}, y(t) \in \mathbb{R}^{m}, A \in \mathbb{R}^{n \times n}, B \in \mathbb{R}^{n \times m}, C \in \mathbb{R}^{m \times n}, D \in \mathbb{R}^{m \times m}$. The vector field $f$ : $\mathbb{R}^{n} \rightarrow \mathbb{R}^{n}$ is assumed to be an admissible nonlinearity.

Initial conditions for Eqs. (14) and (15) are specified by a vector $x(0) \in \mathbb{R}^{n}$ together with a function $y_{0}$ in $C\left([-\tau, 0], \mathbb{R}^{n}\right)$. We write $\left\|x(0), y_{0}\right\|$ for $\max \left(\|x(0)\|_{2},\left\|y_{0}\right\|_{\infty}\right)$ where $\|\cdot\|_{2}$ is the usual Euclidean norm on $\mathbb{R}^{n}$ and $\|\cdot\|_{\infty}$ is the $l_{\infty}$ norm on $C\left([-\tau, 0], \mathbb{R}^{n}\right)$. Under our assumptions on the vector field $f$, the origin is always an equilibrium point of Eqs. (14) and (15). We recall the following stability definitions from [20].

Definition 4.1. The origin is a stable equilibrium of Eqs. (14) and (15) if for any $\varepsilon>0$, there exists some $\delta>0$ such that $\left\|x(0), y_{0}\right\|<\delta$ implies that $\left\|x(t), y_{t}\right\|<\varepsilon$ for all $t \geq 0$. It is said to be asymptotically stable if it is stable and, in addition, $\left\|x(t), y_{t}\right\| \rightarrow 0$ as $t \rightarrow \infty$ for all initial conditions $x(0), y_{0}$.

Our primary concern throughout is with asymptotic stability. In an abuse of notation, we shall refer to the stability of the system (14) and (15) rather than the stability of the equilibrium at the origin.

The system (14) and (15) is said to be absolutely stable if its zero solution is asymptotically stable for every admissible nonlinearity and every $\tau \geq 0$.

Remark. As noted in [20], the second equation (15) can be viewed as a separate system in which $y_{t}$ is the state and $x(t)$ acts as an input. Moreover, it is clear that for the overall system (14) and (15) to be asymptotically stable, the system described by Eq. (15) must be input-to-state stable. This immediately implies that Schur-stability of the matrix $D$ is a necessary condition for asymptotic stability of Eqs. (14) and (15).

Furthermore, if Eqs. (14) and (15) are absolutely stable, then they are asymptotically stable when $f_{i}\left(x_{i}\right)=x_{i}$ for all $i$ and $\tau=0$. It follows immediately that the matrix

$$
A+B(I-D)^{-1} C
$$

must be Hurwitz.

Our next result considers a coupled differential-difference system with Metzler and nonnegative system matrices and shows that in this case the last condition above is sufficient for the existence of a linear $\mathrm{L}-\mathrm{K}$ functional.

Theorem 4.1. Consider the system (14) and (15). Assume that A is a Metzler matrix and that B, $C, D$ are nonnegative matrices. Furthermore, assume that D is Schur-Cohn stable and that 
Eq. (16) is Hurwitz stable. Then there exist positive constants $\mu_{i}, \nu_{i}, 1 \leq i \leq n$, such that

$$
V=\sum_{i=1}^{n} \nu_{i}\left|x_{i}(t)\right|+\sum_{i=1}^{n} \mu_{i} \int_{t-\tau}^{t}\left|y_{i}(z)\right| d z
$$

is a L-K functional for the system defined by Eqs. (14) and (15).

Proof. As $D$ is a Schur-Cohn matrix, there exists some vector $v \gg 0$ with $(D-I)^{T} v \ll 0$. Furthermore, it follows that $I-D$ is an M-matrix and hence has a non-negative inverse. Therefore, the matrix $B(I-D)^{-1} C$ is also non-negative and $A+B(I-D)^{-1} C$ is Metzler. By assumption, this last matrix is also Hurwitz and thus we can choose some $\nu \gg 0$ such that

$$
\left(A+B(I-D)^{-1} C\right)^{T} \nu \ll 0 .
$$

Next set

$$
\mu=\left(I-D^{T}\right)^{-1} B^{T} \nu+\varepsilon v
$$

where $\varepsilon$ is a positive constant. It follows readily from Eq. (19) that

$$
B^{T} \nu+(D-I)^{T} \mu=\varepsilon(D-I)^{T} v \ll 0
$$

for any $\varepsilon>0$. It also follows from Eq. (19) that

$$
A^{T} \nu+C^{T} \mu=\left(A+B(I-D)^{-1} C\right)^{T} \nu+\varepsilon C^{T} v .
$$

From Eq. (18), we see that we can choose $\varepsilon>0$ small enough to ensure that

$$
A^{T} \nu+C^{T} \mu \ll 0
$$

Next note that the Dini upper-right derivative of the functional (17) satisfies the inequality

$$
\partial^{+} V \leq \sum_{j=1}^{n}\left|f_{j}\left(x_{j}(t)\right)\right|\left(\sum_{i=1}^{n} \nu_{i} a_{i j}+\sum_{i=1}^{n} \mu_{i} c_{i j}\right)+\sum_{j=1}^{n}\left|y_{j}(t-\tau)\right|\left(\sum_{i=1}^{n} \nu_{i} b_{i j}+\sum_{i=1}^{n} \mu_{i} d_{i j}-\mu_{j}\right) \text {. }
$$

We can write the right-hand side of Eq. (22) as

$$
|f(x)|^{T}\left(A^{T} \nu+C^{T} \mu\right)+|y(t-\tau)|^{T}\left(B^{T} \nu+(D-I)^{T} \mu\right) .
$$

From Eq. (21), it follows that we can find some $\beta>0$ such that $A^{T} \nu+C^{T} \mu \leq-\beta e$ where $e=(1,1, \ldots, 1)^{T}$. Combining this with Eqs. (20) and (23) yields

$$
\partial^{+} V \leq-\beta \sum_{j=1}^{n}\left|f_{j}\left(x_{j}(t)\right)\right|
$$

It now follows immediately from Theorem 3 of [20] that Eq. (17) is a L-K functional for the system (14) and (15), and that the system is absolutely stable as claimed.

Example 4.1. Consider the system (14) and (15) with matrices given by

$$
\begin{aligned}
& A=\left(\begin{array}{cc}
-4 & 1 \\
1 & -2
\end{array}\right), \quad D=\left(\begin{array}{cc}
1 / 2 & 0 \\
0 & 3 / 4
\end{array}\right), \\
& B=C=\left(\begin{array}{ll}
1 / 2 & 1 / 4 \\
1 / 4 & 1 / 3
\end{array}\right) .
\end{aligned}
$$


Clearly, $D$ is Schur-Cohn; moreover, it can be easily verified that $A+B(I-D)^{-1} C$ is Hurwitz. Thus Theorem 4.1 implies that Eqs. (14) and (15) are absolutely stable and admit an L-K functional of the form (17).

If we combine Theorem 4.1 with the remark preceding it, we obtain the following result.

Corollary 4.1. Consider the system described by Eqs. (14) and (15). Assume that A is a Metzler matrix, B, C, D are non-negative matrices, and D is Schur-Cohn stable. The system (14) and (15) is absolutely stable if and only if the matrix (16) is Hurwitz stable.

\subsection{Switched systems}

We next consider the switched coupled system

$$
\begin{aligned}
& \dot{x}=A^{(\sigma)} f(x(t))+B^{(\sigma)} y(t-\tau), \\
& y(t)=C^{(\sigma)} f(x(t))+D^{(\sigma)} y(t-\tau)
\end{aligned}
$$

and the corresponding family of subsystems

$$
\begin{aligned}
& \dot{x}=A^{(s)} f(x(t))+B^{(s)} y(t-\tau), \\
& y(t)=C^{(s)} f(x(t))+D^{(s)} y(t-\tau), \quad s=1, \ldots, N .
\end{aligned}
$$

It is not too difficult to adapt the argument of Theorem 4.1 to derive the following result.

Theorem 4.2. Consider the switched coupled system (24). Assume that $A^{(s)}$ is a Metzler matrix, and that $B^{(s)}, C^{(s)}, D^{(s)}$ are nonnegative matrices for $s \in\{1, \ldots, N\}$. Further assume that there exists a vector $v \gg 0$ such that

$$
D^{(s)^{T}} v \ll v \text { for } s=1, \ldots, N \text {. }
$$

If there exists a vector $\nu \gg 0$ satisfying

$$
\left(A^{(s)}+B^{(r)}\left(I-D^{(r)}\right)^{-1} C^{(s)}\right)^{T} \nu \ll 0, \quad s, r=1, \ldots, N,
$$

then we can choose positive real numbers $\mu_{1}, \ldots, \mu_{n}$ such that Eq. (17) is a common $L-K$ functional for the family (25) and the system (24) is absolutely stable.

Proof. For $\varepsilon>0$, define

$$
\mu=\max _{r}\left\{\left(I-D^{(r)}\right)^{-T} B^{(r)^{T}} \nu\right\}+\varepsilon v,
$$

where the maximum is again taken componentwise. It can be immediately verified by direct calculation that

$$
B^{(r)^{T}} \nu+\left(D^{(r)}-I\right)^{T} \mu \leq \varepsilon\left(D^{(r)}-I\right)^{T} v \ll 0
$$

for $r=1, \ldots, N$. On the other hand, it follows from our choice of $\nu$ that for $s, r=1, \ldots, N$,

$$
A^{(s)^{T}} \nu+C^{(s)^{T}}\left(I-D^{(r)}\right)^{-T} B^{(r)}{ }^{T} \nu \ll 0 .
$$

Taking the elementwise maximum over $r$, it now follows that we can choose $\varepsilon>0$ sufficiently small to ensure that

$$
A^{(s)^{T}} \nu+C^{(s)^{T}} \mu \leq-\beta e
$$

for some $\beta>0$. 
If we differentiate the functional (17) with respect to the sth subsystem from the family (25), we obtain

$$
\partial^{+} V \leq|f(x(t))|^{T}\left(A^{(s)^{T}} \nu+C^{(s)^{T}} \mu\right)+|y(t-\tau)|^{T}\left(B^{(s)^{T}} \nu+\left(D^{(s)}-I\right)^{T} \mu\right) .
$$

It follows from Eqs. (28) and (29) that

$$
\partial^{+} V \leq-\beta \sum_{i=1}^{n}\left|f_{i}\left(x_{i}\right)\right|
$$

The result now follows immediately.

In the case where the switching only occurs in the state $x(t)$, so that both $B$ and $D$ are fixed, the conditions of the previous result can be relaxed somewhat.

Corollary 4.2. Consider the system (24) and assume that $B^{(s)}=B, D^{(s)}=D$, where $B$, $D$ are nonnnegative and $D$ is Schur-Cohn for $s=1, \ldots, N$. Furthermore, suppose that $A^{(s)}$ is a Metzler matrix, and that $C^{(s)}$ is nonnegative. If there exists a positive vector $\nu$ satisfying

$$
\left(A^{(s)}+B(I-D)^{-1} C^{(s)}\right)^{T} \nu \ll 0, \quad s=1, \ldots, N,
$$

the system (24) is absolutely stable, and there exists a common $L-K$ functional of the form (17) for the family (25).

Remark. As in Section 3, the results of this section can be extended to coupled systems with several delays.

\section{Neutral type systems}

In this section, we present a preliminary result on the stability of neutral systems derived using the techniques of the previous sections.

Consider the switched linear neutral delay system

$$
\dot{x}(t)-D \dot{x}(t-\tau)=A^{(\sigma)} x(t)+G^{(\sigma)} x(t-\tau)
$$

and the corresponding family of subsystems

$$
\dot{x}(t)-D \dot{x}(t-\tau)=A^{(s)} x(t)+G^{(s)} x(t-\tau), \quad s=1, \ldots, N .
$$

Here $x \in \mathbb{R}^{n}, A^{(s)}, G^{(s)}, D$ are constant matrices in $\mathbb{R}^{n \times n}$ and $\tau$ is a constant nonnegative delay. In the stability analysis of neutral systems, it is usual to assume that the operator on the derivative is stable. In our context, this amounts to assuming that the matrix $D$ is Schur-Cohn stable. We make this assumption from here on.

It is known, see for example [21], that the systems in the family (31) can be transformed into the family of coupled delay differential and continuous time difference systems:

$$
\dot{y}(t)=A^{(s)} y(t)+B^{(s)} x(t-\tau), \quad x(t)=y(t)+D x(t-\tau),
$$

where $B^{(s)}=A^{(s)} D+G^{(s)}$.

With this in mind, it is possible to analyse the stability of the system (30) using the results of the previous section. However, we shall use results presented in [1] to provide a sufficient condition for the systems (31) to have a common L-K functional. Note that, in keeping with a standard approach to neutral systems, the systems in the family (31) can be rewritten as follows:

$$
\dot{x}(t)-D \dot{x}(t-\tau)=A^{(s)}(x(t)-D x(t-\tau))+B^{(s)} x(t-\tau),
$$

for $s=1, \ldots, N$. Here $B^{(s)}=A^{(s)} D+G^{(s)}$. 
In contrast to the previous sections, we will not assume that the above systems are positive. In fact, characterising a positive neutral system is not straightforward, and even if we assume that the matrices $A^{(s)}$ are Metzler, and $D, G^{(s)}$ are nonnegative, then it does not necessarily follow that $B^{(s)}$ is nonnegative.

Instead, we use properties of Metzler and nonnegative matrices in an indirect way. We construct the auxiliary matrices $\tilde{A}^{(s)}, \tilde{B}^{(s)}, \tilde{D}$ whose entries are given by

$$
\begin{aligned}
& \tilde{a}_{i i}^{(s)}=a_{i i}^{(s)}, \quad \tilde{a}_{i j}^{(s)}=\left|a_{i j}^{(s)}\right| \text { for } i \neq j, \\
& \tilde{b}_{i j}^{(s)}=\left|b_{i j}^{(s)}\right|, \quad \tilde{d}_{i j}=\left|d_{i j}\right| .
\end{aligned}
$$

With this notation, we have the following result.

Theorem 5.1. Consider the system (30). Suppose that $\tilde{D}$ is a Schur-Cohn stable matrix, and that there exists a positive vector $\nu=\left(\nu_{1}, \ldots, \nu_{n}\right)^{T}$ satisfying

$$
\left(\tilde{A}^{(s)}+\tilde{B}^{(r)}(I-\tilde{D})^{-1}\right)^{T} \nu \ll 0, \quad s, r=1, \ldots, N .
$$

Then the switched system (30) is asymptotically stable for arbitrary admissible switching law and for any $\tau \geq 0$.

Proof. Choose some vector $v \gg 0$ such that $(\tilde{D}-I) v \ll 0$ and for $\varepsilon>0$, define

$$
\mu=\max _{s}\left\{\left(\tilde{B}^{(s)}(I-\tilde{D})^{-1}\right)^{T} \nu\right\}+\varepsilon \nu .
$$

As in the proofs in the last two sections, it can be readily checked that

$$
\left(\tilde{B}^{(s)}\right)^{T} \nu+(\tilde{D}-I)^{T} \mu \ll 0
$$

for all $s$ and that by choosing $\varepsilon>0$ sufficiently small, we can ensure that

$$
\left(\tilde{A}^{(s)}\right)^{T} \nu+\mu \leq-\beta e
$$

for some $\beta>0$.

Now consider a $\mathrm{L}-\mathrm{K}$ functional in the form

$$
V=\sum_{i=1}^{n} \nu_{i}\left|x_{i}(t)-\sum_{k=1}^{n} d_{i k} x_{k}(t-\tau)\right|+\sum_{j=1}^{n} \mu_{j} \int_{t-\tau}^{t}\left|x_{j}(z)\right| d z .
$$

The Dini upper-right derivative along the sth subsystem of Eq. (30) of this functional satisfies

$$
\begin{gathered}
\partial^{+} V \leq \sum_{j=1}^{n}\left|x_{j}(t)-\sum_{k=1}^{n} d_{j k} x_{k}(t-\tau)\right|\left(\sum_{i=1}^{n} \nu_{i} \tilde{a}_{i j}^{(s)}+\mu_{j}\right) \\
+\sum_{j=1}^{n}\left|x_{j}(t-\tau)\right|\left(\sum_{i=1}^{n} \nu_{i} \tilde{b}_{i j}^{(s)}+\sum_{i=1}^{n} \mu_{i} \tilde{d}_{i j}-\mu_{j}\right) .
\end{gathered}
$$

It follows from the first part of the proof that

$$
\partial^{+} V \leq-\beta \sum_{j=1}^{n}\left|x_{j}(t)-\sum_{k=1}^{n} d_{j k} x_{k}(t-\tau)\right| .
$$

The result now follows from Theorem 9.8.4 of [1]. 


\section{Switched nonlinear difference systems with time-delay}

In this section, we briefly note that results on linear functionals similar to those given in Section 3 can also be derived for discrete-time systems. As noted in [22], the systems we consider here arise in applications such as digital filtering.

As in Section 3, we first consider the single delay case in the interest of notational simplicity and clarity.

\subsection{A single delay}

Consider a switched system of the form

$$
x(k+1)=A^{(\sigma)} f(x(k))+B^{(\sigma)} f(x(k-m)),
$$

and the corresponding family of subsystems

$$
x(k+1)=A^{(s)} f(x(k))+B^{(s)} f(x(k-m)),
$$

for $s=1, \ldots, N$. Here, $A^{(s)}, B^{(s)}$ are nonnegative matrices and $m$ is a nonnegative integer delay. The nonlinearities $f(x)=\left(f_{1}\left(x_{1}\right), \ldots, f_{n}\left(x_{n}\right)\right)^{T}$ are continuous for $x \in \mathbb{R}^{n}$ and satisfy Eq. (2). In addition, in this section we will assume that

$$
\left|f_{i}\left(x_{i}\right)\right| \leq\left|x_{i}\right|, \quad i=1, \ldots, n .
$$

We denote by $x_{k}$ the state of the delayed system given by $x_{k}=(x(k), x(k-1), \ldots, x(k-m))^{T}$. Theorem 6.1. Let $A^{(s)}, B^{(s)}$ be nonnegative matrices for $s=1, \ldots, N$. Assume that there exists a vector $\nu \gg 0$ satisfying the inequalities

$$
\left(A^{(s)}+B^{(r)}-I\right)^{T} \nu \ll 0, \quad s, r=1, \ldots, N .
$$

Then there exist positive numbers $\mu_{1}, \ldots, \mu_{n}$ such that

$$
V=\sum_{i=1}^{n} \nu_{i}\left|x_{i}(k)\right|+\sum_{i=1}^{n} \mu_{i} \sum_{l=1}^{m}\left|f_{i}\left(x_{i}(k-l)\right)\right|
$$

defines a common $L-K$ functional for the systems (34).

Proof. As our system is discrete-time, we consider the difference $\Delta V=V\left(x_{k+1}\right)-V\left(x_{k}\right)$ of the functional (37) with respect to the system (34) for some $s \in\{1, \ldots, N\}$. Using Eqs. (34) and (35), we can show by direct calculation that

$$
\Delta V \leq \sum_{j=1}^{n}\left|f_{j}\left(x_{j}(k)\right)\right|\left(\sum_{i=1}^{n} \nu_{i} a_{i j}^{(s)}-\nu_{j}+\mu_{j}\right) \sum_{j=1}^{n}\left|f_{j}\left(x_{j}(k-m)\right)\right|\left(\sum_{i=1}^{n} \nu_{i} b_{i j}^{(s)}-\mu_{j}\right) .
$$

It is clear from Eq. (38) that if we can find a positive vector $\mu \gg 0$ such that

$$
\left(A^{(s)}-I\right)^{T} \nu+\mu \ll 0, \quad B^{(s)^{T}} \nu-\mu \ll 0, \quad s=1, \ldots, N,
$$

then Eq. (37) will define a common L-K functional for the family of systems (34).

To see that such a vector $\mu$ must exist, note that as there are only finitely many inequalities in Eq. (36), there exists some positive vector $w \gg 0$ with

$$
\left(A^{(s)}-I\right)^{T} \nu+\left(B^{(r)}\right)^{T} \nu \ll-w
$$


for $s, r$ in $\{1, \ldots, N\}$. Define $d=\max _{r}\left\{\left(B^{(r)}\right)^{T} \nu\right\}$ as the componentwise maximum of the vectors $\left(B^{(r)}\right)^{T} \nu$. It is now easy to verify that $\mu=d+w / 2$ satisfies Eq. (39) and hence for this choice of $\mu$, Eq. (37) defines a L-K functional for Eq. (33) as claimed.

Example 6.1. Consider the system (33) with two constituent systems whose matrices are given by

$$
\begin{aligned}
& A^{(1)}=\left(\begin{array}{cc}
1 / 2 & 0 \\
1 / 4 & 1 / 4
\end{array}\right), \quad B^{(1)}=\left(\begin{array}{cc}
1 / 8 & 0 \\
0 & 1 / 12
\end{array}\right) ; \\
& A^{(2)}=\left(\begin{array}{cc}
2 / 5 & 1 / 10 \\
0 & 3 / 10
\end{array}\right), \quad B^{(2)}=\left(\begin{array}{cc}
1 / 5 & 0 \\
0 & 1 / 10
\end{array}\right) .
\end{aligned}
$$

In this case, we need to find a vector $\nu \gg 0$ such that $\left(A^{(1)}+B^{(1)}-I\right)^{T} \nu \ll 0$, $\left(A^{(1)}+B^{(2)}-I\right)^{T} \nu \ll 0,\left(A^{(2)}+B^{(1)}-I\right)^{T} \nu \ll 0,\left(A^{(2)}+B^{(2)}-I\right)^{T} \nu \ll 0$. It is not difficult to check that the vector $\nu=(1,1)^{T}$ will suffice in this instance. Thus, the system (33) is absolutely stable and admits an $\mathrm{L}-\mathrm{K}$ functional of the form (37). In fact, we can choose the vector $w=(1 / 25,1 / 4)^{T}$ and find that $d=(1 / 125,1 / 40)^{T}$. Therefore, setting $\mu=(7 / 250,6 / 40)^{T}$ we obtain the functional whose existence is guaranteed by the theorem.

\subsection{Several delays}

Finally, for this section, consider the family of subsystems

$$
x(k+1)=A^{(s)} f(x(k))+B_{1}^{(s)} f(x(k-1))+\cdots+B_{m}^{(s)} f(x(k-m)),
$$

for $s=1, \ldots, N$. Here $A^{(s)}, B_{1}^{(s)}, \ldots, B_{m}^{(s)}$ are nonnegative matrices for $1 \leq s \leq N$, and the nonlinearities $f(x)=\left(f_{1}\left(x_{1}\right), \ldots, f_{n}\left(x_{n}\right)\right)^{T}$ satisfy the conditions (2) and (35).

We note that it is relatively straightforward to adapt the argument used in the proof of Theorem 6.1 to obtain the following result for systems with several delays.

Theorem 6.2. Assume that there exists a positive vector $\nu \gg 0$ satisfying the inequalities

$$
\left(A^{(s)}+B_{1}^{\left(r_{1}\right)}+\cdots+B_{m}^{\left(r_{m}\right)}-I\right)^{T} \nu \ll 0,
$$

for $s, r_{1}, \ldots r_{m}=1, \ldots, N$. Then there exist positive constants $\mu_{l j}$, such that

$$
V=\sum_{i=1}^{n} \nu_{i}\left|x_{i}(k)\right|+\sum_{j=1}^{n} \sum_{l=1}^{m} \mu_{l j}\left(\left|f_{j}\left(x_{j}(k-1)\right)\right|+\cdots+\left|f_{j}\left(x_{j}(k-l)\right)\right|\right)
$$

defines a common $L-K$ functional for the family (40).

\section{Conclusions}

We have presented a set of results concerning Lyapunov-Krasovski functionals and absolute stability for various classes of nonlinear, switched systems with time-delay. Specifically, we have described sufficient conditions for absolute stability of the system class considered in [18] that relax the requirements of this previous paper. We have extended this analysis to systems described by coupled differential-difference equations and neutral systems. We have also briefly noted that corresponding results can be obtained for discrete-time systems. There are several possibilities for developing the work described here. In particular, it would be of interest to 
investigate whether our analysis can be adapted to the design of stabilising switching sequences [23] or to address questions of controller synthesis such as those considered in $[7,8]$.

\section{References}

[1] J.K. Hale, S.M. Verduyn Lunel, Introduction to Functional Differential Equations, Springer-Verlag, New York, 1993.

[2] V.L. Kharitonov, Robust stability analysis of time-delay systems: a survey, Annu. Rev. Control 23 (1999) 185-196.

[3] V.L. Kharitonov, Lyapunov functionals and matrices, Annu. Rev. Control 34 (2010) 13-20.

[4] L. Farina, S. Rinaldi, Positive Linear Systems. Theory and Applications, Pure and Applied Mathematics, John Wiley \& Sons Inc., New York, 2000.

[5] T. Kaczorek, Positive 1D and 2D Systems, Springer-Verlag, London, 2002.

[6] F. Knorn, O. Mason, R. Shorten, On copositive linear Lyapunov functions for sets of linear positive systems, Automatica 45 (8) (2009) 1943-1947.

[7] S. Li, Z. Xiang, H. Reza Karimi, Stability, L1-gain and control synthesis for positive switched systems with timevarying delay, Nonlinear Anal.: Hybrid Syst. 9 (2013) 9-17.

[8] S. Li, Z. Xiang, H. Reza Karimi, Stability and L1-gain controller design for positive switched systems with mixed time-varying delays, Appl. Math. Comput. 222 (2013) 507-518.

[9] A.Yu. Aleksandrov, A.V. Platonov, On absolute stability of one class of nonlinear switched systems, Autom. Remote Control 69 (7) (2008) 1101-1116.

[10] W. Xiang, J. Xiao, Stability analysis and control synthesis of switched impulsive systems, Int. J. Robust Nonlinear Control 22 (2012) 1440-1459.

[11] W. Xiang, J. Xiao, Asymptotic stability, $l_{2}$ gain boundness analysis and control synthesis for switched systems: a switching frequency approach, Int. J. Adapt. Control Signal Process. 26 (2012) 350-373.

[12] W. Xiang, J. Xiao, Stabilization of switched continuous-time systems with all modes unstable via dwell time switching, Automatica 50 (3) (2014) 940-945.

[13] E. Fornasini, M.E. Valcher, Stability and stabilizability criteria for discrete-time positive switched systems, IEEE Trans. Autom. Control 57 (5) (2012) 1208-1221.

[14] F. Blanchini, P. Colaneri, M.E. Valcher, Co-positive Lyapunov functions for the stabilization of positive switched systems, IEEE Trans. Autom. Control 57 (12) (2012) 3038-3050.

[15] S.K. Persidskii, Problem of absolute stability, Autom. Remote Control 12 (1969) 1889-1895.

[16] W.M. Haddad, V. Chellaboina, Stability theory for nonnegative and compartmental dynamical systems with time delay, Syst. Control Lett. 51 (2004) 355-361.

[17] A.Yu. Aleksandrov, Y. Chen, A.V. Platonov, L. Zhang, Stability analysis for a class of switched nonlinear systems, Automatica 47 (10) (2011) 2286-2291.

[18] Y. Sun, L. Wang, On stability of a class of switched nonlinear systems, Automatica 49 (1) (2013) 305-307.

[19] D.M. Stipanovic, D.D. Siljak, Stability of polytopic systems via convex $M$-matrices and parameter-dependent Lyapunov functions, Nonlinear Anal. 40 (1-8) (2000) 589-609.

[20] K. Gu, Y. Liu, Lyapunov-Krasovskii functional for uniform stability of coupled differential-functional equations, Automatica 45 (3) (2009) 798-804.

[21] P. Pepe, On the asymptotic stability of coupled delay differential and continuous time difference equations, Automatica 41 (1) (2005) 107-112.

[22] E. Kaszkurewicz, A. Bhaya, Matrix Diagonal Stability in Systems and Computation, Birkhäuser, Boston, Basel, Berlin, 2000.

[23] A. Zappavigna, P. Colaneri, J. C. Geromel, R. Shorten, Dwell time analysis for continuous-time switched linear positive systems, in: Proceedings of the American Control Conference, 2010, pp. 6256-6261. 